\section{ARTIFICIAL METALLOENZYMES}

\section{Encoding copper catalysts}

Chem. Sci. http://dx.doi.org/10.1039/

c4sc01525h (2014)

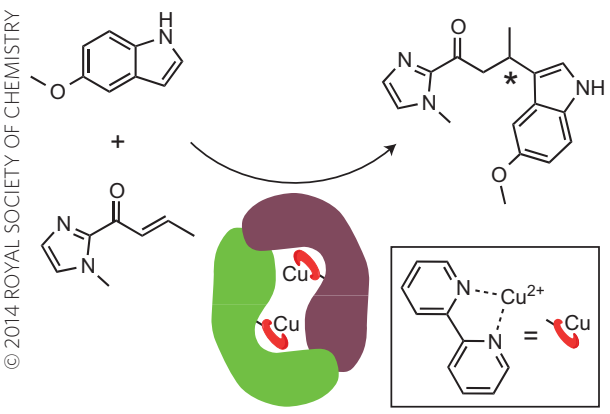

Artificial metalloenzymes offer the opportunity to marry the versatility of transition metal catalysts with the very high activity and selectivity provided by enzymatic scaffolds. However, designing new metalloenzymes is complicated because transition metals typically require multiple donor atoms to form a stable complex. A single natural amino acid cannot normally provide more than one donor atom and designing new proteins that fold to form a three-dimensional metal binding site is not currently possible. Incorporating unnatural amino acids that contain side-chains capable of binding transition metals into a protein's amino acid sequence offers one solution to this problem.

To achieve this aim, a team led by Gerard Roelfes at the University of Groningen used the 'amber stop codon suppression' method to site-specifically insert an unnatural amino acid into a protein called 'Lactoccocal multidrug resistance Regulator' (LmrR). In this approach the amber stop codon and an orthogonal tRNA/aminoacyl-tRNA synthase pair are used to incorporate an unnatural amino acid at the position within the protein designated by the amber stop codon. The team used an unnatural amino acid with a 2,2'-bipyridine moiety on its side-chain to create variants of LmrR that were capable of binding $\mathrm{Cu}^{2+}$ ions and were observed to form dimers.
Next, the team investigated whether these artificial metalloproteins were capable of catalysing a Friedel-Crafts alkylation. Variants of LmrR containing bound copper were shown to not only catalyse the reaction but also provide a modest enantiomeric excess. Comparative experiments using a simple copper complex as the catalyst showed no enantiomeric selectivity. To optimize the catalytic properties of the metalloenzyme, the team carried out a mutagenesis study in which amino acid residues surrounding the active site were swapped for alternative amino acids. Successive rounds of mutagenesis produced variants that improved both the yield and the enatioselectivity of the reaction.

ON-SURFACE ASSEMBLY

\section{The squeezed middle}

Angew. Chem. Int. Ed. http://dx.doi.org/10.1002/ anie.201406246 (2014)

Surfaces provide an interface on which molecules can order themselves over a longer range than they might achieve in

\title{
2014 NOBEL PRIZE IN CHEMISTRY
}

\section{No limit}

"Ingenious." That is how the Royal Swedish Academy of Sciences described the work of Eric Betzig of the Howard Hughes Medical Institute, Virginia, USA; Stefan W. Hell of the Max Planck Institute for Biophysical Chemistry, Göttingen, Germany; and W. E. Moerner from Stanford University, USA, who have shared the 2014 Nobel Prize in Chemistry. The prize was awarded for the development of fluorescence techniques with nanometre 'superresolution' that have enabled scientists to observe chemistry occurring in unprecedented detail. These techniques sidestep a fundamental physical law first described by Ernst Abbe in the late nineteenth century - that limits standard optical microscopy to a resolution of roughly $200 \mathrm{~nm}$.

Working separately, Moerner (pictured left) and Betzig (pictured middle) laid the foundations for 'single-molecule spectroscopy'. Moerner, as well as being the first person to measure the absorption spectrum of a single fluorophore molecule in 1989, discovered a controllable variant of green fluorescent protein (GFP) in 1997. This GFP could be switched on with

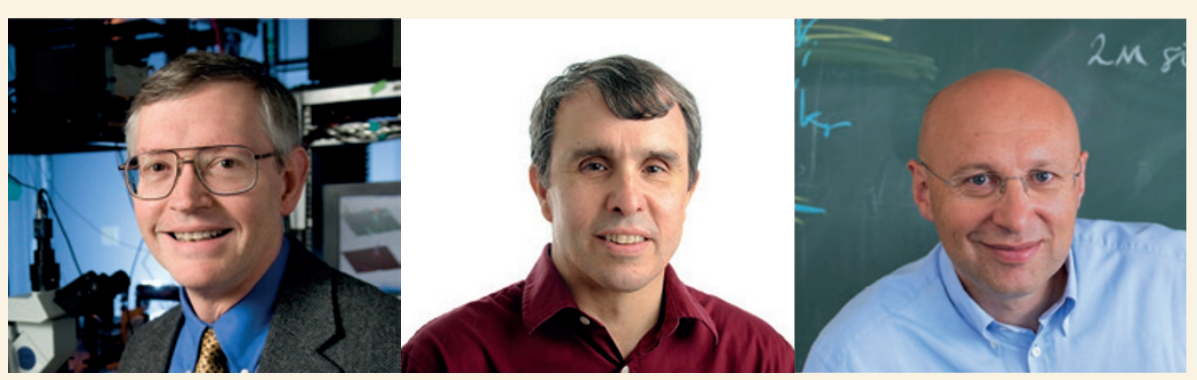

IMAGES LEFT-RIGHT: (C LA CICERO/STANFORD UNIVERSITY; (C) MATT STALEY/HOWARD HUGHES MEDICAL INSTITUTE; (C) BERND SCHULLER/MAX PLANCK INSTITUTE FOR BIOPHYSICAL CHEMISTRY

light of a specific wavelength and would intermittently fluoresce for a period of time before switching off - it could then be reactivated by a second wavelength of light. Using similar proteins, Betzig reported the technique of 'photo-activated localization microscopy' (PALM) in 2006. This method uses a weak laser to switch on the fluorescence of a fraction of the fluorophores in a sample, which are almost always farther apart than the $200 \mathrm{~nm}$ diffraction limit. They are imaged before the fluorescence is turned off, and when the process is repeated a different set of fluorophores light up. This process is carried out many times and a composite of the images gives a high-resolution picture of the positions of the fluorophores.

Hell (pictured right) developed a technique known as stimulated emission depletion (STED) microscopy. It works by using a laser to stimulate fluorescence in a sample of labelled molecules and a second laser beam that prevents fluorescence from a doughnut-shaped region around the point of interest. Only the fluorescence from the central area is detected. The microscope can then be swept across the sample, nanometre-by-nanometre, until a large picture is built up. Hell first published the concept in 1994, but it was not demonstrated experimentally until 2000. 